\title{
PERIODIC NOISE SUPPRESSION TECHNIQUES APPLIED TO REMOTE SENSING IMAGES
}

\author{
C.R.Souza Filho ${ }^{1} \&$ A.Dinniss ${ }^{2}$
}

KEY-WORDS: Noise, fourier transforms, image restoration.

SOUZA FILHO, C.R.; DINNISS, A. (1997) Periodic noise suppression techniques applied to remote sensing images. Bol.lG-USP, Sér.Cient., 28:23-61.

\begin{abstract}
The image data collected by the JERS-1 OPS, SPOT and AVHRR systems are affected by a variety of periodic (coherent) noise problems, which can be severe in many cases. This paper examines some of these defects and also describes and evaluates a series of methodologies to recover partially or totally the information contained in these data by means of: (i) filtering in the spatial domain (convolution filters), (ii) Principal Components Analysis (PCA) and (iii) filtering in the frequency domain (using Fast Fourier Transforms).

Filtering in the spatial domain using relatively small convolution filters can be successfully applied to tackle elementary periodic noise problems. However, variations of theoretically efficient kernels experimented in this study were only able to minimise the effect of such complex noise structures at the expense of significant modification or complete loss of important raw image data.

Principal Component Analysis transforms the data so that the noise component is cast into one or more of the high-order principal components. Our experiments show that this technique is able to confine the noise in the higher order PCs, but a significant amount of residual noise was present in the low-order components.

Filtering in the frequency domain proved to be a suitable technique to achieve image restoration whilst preserving most of the raw scene information intact. Conventional Fourier operators like the Notch Filter and the Low-pass Elliptically Symmetric Filter (with a Gaussian-shaped intensity profile) can tackle the problems with some noisy images quite reasonably. However, such filters are unable to recover images on which the amplitude of the noise varies heterogeneously producing a series of impulses from low to high frequencies. Both the Zonal Notch Filter (based on the difference of the Fourier spectra of two channels) and the Synergistic Filter (which relies on the convolution theorem) are designed to contour this problem. These are interactive restoration methods that can successfully eliminate or minimise the effects of multiple two-dimensional periodic structures superimposed on both multi-channel and single channel data, respectively.
\end{abstract}

\section{RESUMO}

Dados digitais coletados por diversos sensores orbitais (e.g., JERS-1 OPS, SPOT e AVHRR) são comumente afetados por ruídos periódicos (coerentes). Ruídos periódicos são produzidos por erros de instrumentação e/ou flutuações eletrônicas nos sensores e acarretam problemas no processamento e interpretação de imagens digitais. Para que dados ruidosos possam ser melhor utilizados

\footnotetext{
'Instituto de Geociências/UNICAMP, Campinas, SP, Brasil.

${ }^{2}$ British Geological Survey, Keyworth, Nottingham, United Kingdom.
} 
é essencial que o ruido presente nestes seja atenuado ou eliminado. Este artigo examina os ruidos tipicamente presentes em imagens de sensoriamento remoto, abordando uma série de metodologias alternativas para recuperação parcial ou total da informação contida nestes dados. As metodologias investigadas compreendem: (i) filtragem no dominio espacial (filtros de convolução); (ii) análise por Principais Componentes (APC); e, (iii) filtragens no dominio de frequências, utilizando-se Transformadas de Fourier.

A filtragem no dominio espacial, por meio de filtros de convolução relativamente pequenos e velozes, pode ser aplicada com sucesso na minimização de ruídos periódicos em imagens de sensoriamento remoto. Entretanto, a grande maioria dos filtros espaciais clássicos disponiveis mostra-se incapaz de atenuar ruidos complexos sem alterar em demasia o sinal da imagem.

A análise por Principais Componentes (PCs), por sua vez, realiza uma transformação nos dados de forma que o ruido é alocado em uma ou mais PCs de maior ordem. Nossos experimentos indicam que muito embora esta técnica seja capaz de isolar boa parte do ruído nas PCs de maior ordem, uma grande quantidade de ruido residual ainda permanece nas primeiras PCs.

A filtragem no dominio de frequências, utilizando-se operadores Fourier, constituise na mais poderosa ferramenta para a filtragem de ruidos periódicos e recuperação de imagens de sensoriamento remoto ruidosas. Neste processo, o ruido periódico pode ser eliminado seletivamente sem perda ou modificação do sinal. Métodos tais como os filtros Fourier sintonizȧveis clássicos (notch filters) e os filtros Fourier passa-baixa elipticamente simétricos (com um perfil de intensidade gaussiano) podem ser utilizados na recuperação deste tipo de dados ruidosos. Entretanto, estes métodos são limitados, principalmente quando os intervalos de frequência associados aos ruidos se superpõem àqueles ocupados pelo sinal da imagem (neste casos, a energia relativa ao ruido mostra-se misturada à energia correspondente ao sinal em regiōes de baixa frequência), o que tipicamente ocorre em imagens de sensoriamento remoto. As técnicas aqui apresentadas, denominadas Filtro Sintonizável Zonal (Zonal Notch Filter - envolvendo a subtração do espectro Fourier de duas bandas espectrais distintas) e o Método Sinergistico (compreendendo variaçōes do uso do teorema da convolução), são técnicas adequadas e capazes de atenuar ou eliminar os efeitos produzidos por ruidos periódicos bidimensionais complexos, superimpostos ao sinal de dados multi-canais e de canal único, respectivamente.

\section{INTRODUCTION}

The past and currently operational satellite and airborne platforms have acquired images that are plagued by periodic noise. These are assembled mainly by sensor failures and instrumentation errors. Noise artefacts can be added to the imagery either erratically (e.g., panchromatic High Resolution Visible (HRV) Système Probatoire de l'Obervation de la Terre (SPOT); Landsat Thematic Mapper (TM)) or continuously (e.g., Japan Earth Resources Satellite (JERS-1) OPtical System (OPS); Advanced Very High Resolution Radiometer (AVHRR)) along the life period of a sensor. Since the human vision is highly sensitive to structured patterns, periodic noise can be particularly distracting and obstructive in the interpretation of remotely sensed imagery. It can also be further enhanced by image processing techniques. Suppression of its effects is imperative in most cases.

The literature lacks of a unique technique capable of analysing and reducing the artefacts observed in several data from multiple sensors - the available noise removal and image restoration methods have been developed to solve specific imagery problems within specific sensors (orbital data: (i) Crippen, 1989 - Landsat TM; (ii) Westin, 1990 - SPOT; (iii) Warren, 1989; Simpson \& Yhann, 1994 - AVHRR; (iv) de Souza Filho et al., 1996 - JERS-1 OPS; airborne data: Rose, 1989 - AVIRIS; Hummer-Miller, 1990 - TIMS; Penteado et al., 1997 - GEOSCAN AMSS MKII). That poses problems for data handling and use of combined imagery datasets.

Techniques to remove noise or to 
suppress its effects within an image can either be applied in the spatial domain (standard Cartesian form) or in the frequency domain (Fourier transform). Both approaches as applied to images derived from multiple sensors, with emphasis on JERS-1 data, are briefly described and discussed here. In the view of its vast potential use, the main objective of this paper centres in developing an optimum method for the cosmetic removal of periodic noise from digital data, despite its source. The alternative method attempted here involves the synergism of standard convolution kernels and Fourier operators. It is presented in a manner that can be customised for most image processing packages, not requiring the complex mathematical background usually employed in the implementation of most available noise removal methods.

\section{REMOTE SENSING NOISY DATA}

The data used in this paper comprise four sub-scenes of highly corrupted JERS-1 OPS, SPOT and AVHRR images. Since they comprehend the most complex noise structures ever seem in commercial satellite data, the JERS-1 OPS data is used throughout the paper to illustrate the relative effectiveness of well-established filtering approaches. SPOT and AVHRR data are also employed to portray the efficacy and hindrance of the synergistic noise removal method. Figures $1 \mathrm{a}, \mathrm{b}, \mathrm{c}$ and $d$ show these originally noisy subscenes of JERS-1 OPS Level-2 channel 8 data of Eritrea (NE Africa), JERS-1 OPS Level-2 channel 6 data of Oman, panchromatic SPOT 3 HVR1 Level $1 \mathrm{~A}$ data of Ghana, and NOAA9 AVHRR channel 3 data of Sicily (Mt Etna).

The image data collected by JERS-1's sensors covering the visible (VIS; channels 1,2), near-infrared (NIR; channel 3-4) and the short wavelength infrared (SWIR; channels 5,6,7,8) regions are affected by severe noise problems. The important narrow SWIR channels show the worst defects. Artefacts originally introduced by the satellite sensors (unprocessed Level 0 data) were exaggerated by the radiometric and geometric correction applied by NASDA (Level 2 data). These defects have a very systematic and specific nature and comprise both across-track and along-track stripping. Some noise structures are related to real features in the scene and distinction between the two in the spatial domain is difficult (Figs. 1a, b). A full description of JERS-1 noise structures can be found in de Souza Filho et al. (1996) and it will not be repeated here.

Periodic to pseudo-periodic noise have been revealed within some of the SPOT 1 (e.g., Westin, 1990 and references therein), SPOT 2 and SPOT 3 (Timothy Minor; personal communication) imagery after pos-launch investigations of the data. Such noise has been documented in panchromatic images from the first (Fig. 1c) and second High Resolution Visible (HRV 1 e 2) where an along-column (along-track) noise occurs. This line-to-line noise is coherent along lines, but the amplitude varies along columns. Similar noise, but of lower amplitude, has also been reported for panchromatic images from HRV1, where a corresponding noise in the perpendicular direction (along lines; across track) was also perceived. As concluded in Westin (1990), in all cases, the dominant period is two pixels, and the amplitude varies slowly over the image. This noise was most pronounced during the first year of operation of the SPOT 1 , subsequently diminished in magnitude and was later only a problem in dark scenes with low dynamic range. Something similar seemed to have occurred with SPOT 2 and 3. CNES recently confirmed that 
(a)

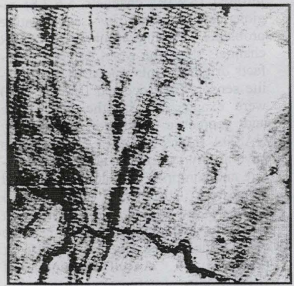

(c)

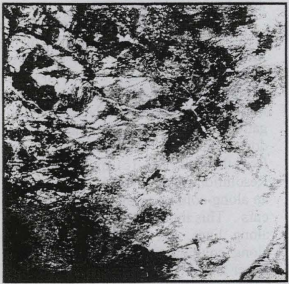

(b)

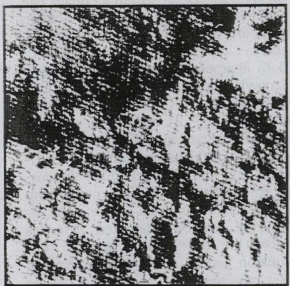

(c)

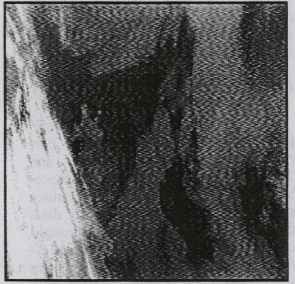

Figure 1 - Noisy sub-scenes of (a) JERS-1 OPS Level-2 channel 8 data of Eritrea (NE Africa); (b) JERS-1 OPS Level-2 channel 6 data of Oman; (c) panchromatic SPOT 3 HVR1 Level 1A data of Ghana (georeferenced); (d) NOAA9 AVHRR channel 3 data of Silicy (Mt Etna).

the SPOT 3 spacecraft, operating since September/1993, suffered an unrecoverable malfunction on November/1996. The SPOT 1, in orbit since February 1986, was reactivated on 9 January 1997, and acquired its first scenes in the real-time acquisition mode, ensuring continuity of service alongside SPOT 2. It is probable that the images as ac- quired by SPOT 1 will continue suffering of the same noise problems reported here.

The channel 3 data of the AVHRR on the NOAA series of satellites (NOAA 6-12) are contaminated by severe instrumentation noise (Waren, 1989). The signal-to-noise ratio varies considerably from image to image and 
its variation between sensors (e.g. NOAA7 versus NOAA 9) can be large (Simpson \& Yhann, 1994). The channel 3 noise is highly directionally dependent. When viewed along each image line (horizontal direction), the noise varies slowly and is almost coherent. Along the image columns (vertical direction), however, the noise varies rapidly and is almost random in nature (Fig. 1d). Simpson \& Yhann (1994) provided a detailed characterisation of channel 3 noise structures and this will not be reproduced further here. In the next sections, we describe and discuss some of the basic and complex techniques used in noise removal approaches in both spatial and frequency domains.

\section{SPATIAL DOMAIN METHODS}

\section{Algebraic}

One of the simplest techniques to remove noise from images is that of image averaging (Castleman, 1979). This technique relies on the production of a cleaned output image by averaging $N$ input channels. Although simple to implement, this technique is best used to remove random noise. Ideally, it should be applied when it is possible to obtain images of a stationary scene (i.e., over the same geographic region and comprising the same waveband). If these images are contaminated by an additive noise source, the average of multiple images can reduce the noise. In the averaging process, the stationary component of the image is unchanged, whereas the noise pattern, different from one image to the next, builds up more slowly in the sum. Our experience shows that remote sensing studies involving temporal analysis can make good use of the amount of available scenes to perform this algebraic operation and successfully reduce eventual random noise.

\section{Convolution filtering}

Modern pipelined processors are capable of performing very fast convolution operations. Consequently, a significant amount of research has focused on the design, implementation, and applications of finite impulse response (FIR) filters for digital image restoration (Strickland \& Aly, 1985).

Convolution filtering is a contextdependent operation that alters the grey level of a pixel based on a weighted average of a kernel of pixels (matrix) centred about the pixel of interest. It involves multiplying individual DN values in an input image by a box filter which contains a matrix of weighted values; the product of this operation is then used to replace the original image DN value at the centre of the matrix. A new output image is produced by moving the matrix over every pixel in the input scene. The basis of the convolution technique has been extensively summarised in the literature (e.g., Drury, 1993), is easily understandable and will not be discussed again here. However, once the convolution basics have been grasped, the problem is the enormous number of possible kernels available. The question is "How to choose the weighting factors appropriate for the noise removal challenge at hand?". An optimal approach usually considers the relation between convolution and Fourier filtering (Gonzalez \& Wintz, 1977). This implies manipulation of data in the frequency domain, thereby defeating the aims of this section. Where frequency domain resources are unavailable, the other possible way to tackle major noise problems involves standard convolution kernels and their variations.

The beauty of convolution filtering is that it is very flexible as both the size and the weightings of the convolution kernel (matrix) can be easily changed. Varying these parameters 
means that the effect of the filter on the output image can be controlled. In practice convolution filters are used to either enhance or suppress edge information (high frequencies) in an image; these are respectively known as high- or low-pass filters. Noise removal by convolution tends to use low pass filters as these suppress the visibility of noise artefacts in an image.

Noise is known to grow with increasing spatial frequencies (Castleman, 1979). If this is correct, its effects can be minimised by averaging the pixel values of an image, causing them to be more homogeneous. Low pass kernels, such as mean filters are designed to perform this sort of matrix algebra. This mean filter replaces the central DN value with the average DN of the surrounding 8 pixels (for a $3 \times 3$ kernel). This has the effect of suppressing any high frequency variations, i.e. those associated with random noise, within a 3 x 3 image window (Castleman, 1979). Mean filters can be composed of as many elements as desired by the user, but usually the following are employed:

\section{KERNEL1 KERNEL 2 KERNEL 3 KERNEL 4}

$$
\frac{1}{9}\left[\begin{array}{lll}
1 & 1 & 1 \\
1 & 1 & 1 \\
1 & 1 & 1
\end{array}\right] \quad \frac{1}{25}\left[\begin{array}{lllll}
1 & 1 & 1 & 1 & 1 \\
1 & 1 & 1 & 1 & 1 \\
1 & 1 & 1 & 1 & 1 \\
1 & 1 & 1 & 1 & 1 \\
1 & 1 & 1 & 1 & 1
\end{array}\right]
$$

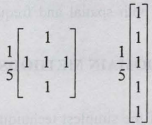

Tests on images corrupted by both periodic and random noise (e.g., JERS1 data) show that this type of filter does suppress some of the random noise but this is achieved at the expense of suppressing high frequency information (such as topographic edges and tonal boundaries). The output image appears slightly blurred. The mean filter fails to minimize the effects of periodic patterns. Broader kernels (such as $5 \times 5$ or larger) can provide a considerable compression of frequencies but at the expense of even larger levels of blurring. The noise observed in channel 3 AVHRR images can be reasonably resolved by convolving the image with a $5 \times 1$ averaging kernel (Kernel 4) (Simpson \& Yhann, 1994); in this case the high frequency variation along each column is reduced, thereby reducing the overall noise in the image. Restored channel 3 images still show, however, a significant amount of remaining low frequency noise.

Trials to concomitantly reduce noise and avoid unsatisfactory blur can be carried out by simply varying the outer weights of a mean matrix. Use of scale factors on the kernels are not suggested as they may significantly modify the statistical integrity of the image data. The three by three filter is the smallest odd kernel size that can be used and gives minimal smoothing. We tested a multitude of kernel variations from the literature in noisy image data collected by a multitude of sensors, and the most efficient are commented on.

All kernels shown above are able to reduce the effects of noise presented by JERS-1, SPOT and AVHRR imagery. Kernel 6 and 8 provided the best overall results. It is important to emphasise, however, that these kernels should be used as a basis for experimentation rather than taken as 'frozen' 
KERNEL 5 KERNEL $6 \quad$ KERNEL 7 KERNEL 8

$$
\left[\begin{array}{lll}
1 & 1 & 1 \\
1 & 2 & 1 \\
1 & 1 & 1
\end{array}\right] \quad\left[\begin{array}{lll}
1 & 1 & 1 \\
1 & 4 & 1 \\
1 & 1 & 1
\end{array}\right] \quad\left[\begin{array}{lll}
1 & 2 & 1 \\
2 & 3 & 2 \\
1 & 2 & 1
\end{array}\right] \quad\left[\begin{array}{lll}
1 & 2 & 1 \\
2 & 4 & 2 \\
1 & 2 & 1
\end{array}\right]
$$

KERNEL 7 KERNEL 8 KERNEL 9

$$
\left[\begin{array}{ccc}
1 & 4 & 1 \\
4 & 12 & 4 \\
1 & 4 & 1
\end{array}\right] \quad\left[\begin{array}{lll}
0 & 1 & 0 \\
1 & 4 & 1 \\
0 & 1 & 0
\end{array}\right] \quad\left[\begin{array}{lll}
0 & 1 & 0 \\
1 & 2 & 1 \\
0 & 1 & 0
\end{array}\right]
$$

designs. That is because noise structures may vary from both channel to channel and scene to scene, and therefore, specific filters may be selected to minimise specific interference patterns. We also noted that although these small kernels can decrease the impression of the noise, they correspond to crude filtering functions with limited control on spatial frequencies. Consequently, noise structures will still be present in the filtered images to a certain degree, even if not causing evident signal obstruction.

An alternative to the mean filter is the median filter (Kernel 3) which replaces the central image DN value with median value derived from the surrounding 8 pixels. Tests show that the median filter removes isolated random noise as well as the mean filter, although less image blur is introduced. Moreover the median filter is capable of dissembling some of the periodic noise effects, it does not remove it.

The convolution kernel operations can be enhanced by the introduction of an algorithm which decides whether a pixel requires replacing or not, depending on its deviation from the surrounding pixel values. If a pixel deviates more than a given threshold value, it is then replaced; otherwise it remains unchanged. Using an algorithm such as this ensures that only the minimal amount of image pixels are changed, thereby helping to preserve image detail. An example of this algorithm is given by Schowengerdt (1983). A similar approach is referred to in the literature as adaptive filtering (e.g., Peli \& Lim, 1982; Eliason \& McEwen, 1990). These filters are designed for best removing speckled noise, bit errors, dropout errors and particular bad lines or columns of data, where the surrounding data is assumed to be valid. A drawback is that this technique relies on the fact that the pixels surrounding a noisy one are themselves good. Unfortunately this is often not the case, particularly with the periodic structures observed in JERS-1, SPOT and AVHRR noisy data, which usually affect contiguous pixels and vary differently along image lines and columns. In such circumstances, adaptive filters will in general replace each pixel with the pixel values averaging along lines only. This cannot reduce the noise impact in any of the data employed here without their significant smoothing and loss of spatial detail.

\section{Principal components analysis Background}

The principal components (PC) transformation is a multivariate statistical technique which selects uncorrelated 
linear combinations (eigenvector loadings) of variables in $\mathrm{n}$-dimensional space in such a way that each successively extracted linear combination, or principal component ( $\mathrm{PC})$, has a smaller variance (Singh \& Harrison, 1985). Its main advantages of reducing data "dimensionality' and decorrelating highly correlated images are well known (Ready \& Wintz, 1973).

The statistical variance in multispectral images is related to the spectral response of various scene attributes and it is also influenced by the statistical dimensionality of the image data. When multispectral image channels are treated as variables and subjected to PC transformation it follows that the ordering of the principal components is influenced both by the spatial abundance of the various surficial materials and by the image statistics. In this case, it is possible to take advantage of the influence of scene statistics, which can be both measured and adjusted, in order to 'force' the transformation to provide information on the spatial distribution and relative abundance of specific image attributes (Loughlin, 1991), these being either useful information about rocks, soils and vegetation or unwanted artefacts such as noise.

Multichannel sets, like the noisy JERS-1 data, when subjected to PC transformation are ordered in a such a way that the first PC contains the maximum possible proportion of the variance in the original data (i.e., interband correlated information), with later components accounting for successively smaller amounts of the remaining variance (interband poorly correlated information). Fundamentally, increasing order PCs have progressively lower signal-to-noise ratios (SNR). In geological remote sensing, this means that lower PC images have less geological variance rather than variance associated with sensor-derived defects and atmos- pheric effects. The noise variance of a scene is then approximated by the lowest eigenvalue and is commonly found in the lowest order PC image under analysis; though Green et al. (1988), Townsend (1984) and Drury \& Hunt (1988) pointed out that the decrease of SNR as a function of increase in the PCs order may not always occur so steadily.

These principles being stated, it becomes clear that in multichannel image analysis, the inclusion of all channels in the PC transformation increases the likelihood that any unwanted data are isolated by the process, provided of course that the noise structures are poorly correlated between channels. If, after the forward transformation, the noise is largely isolated from image signals among the PCs, then it can be removed substantially by setting the noisy $\mathrm{PCs}$ to a constant value prior to the reverse transform back to wavebands.

\section{The forward transformation}

Table 1 lists (1) the image statistics, (2) the variance-covariance matrix, (3) the eigenvalues (which give an indication of decreasing variance in successive principal components) and, (4) the eigenvectors loadings (linear combinations of weighted input images in the $\mathrm{PCs}$ ) of a forward PC transformation based on the variance-covariance ma trix, for a selected set of all seven "raw" JERS-1 OPS channels acquired over Eritrea. Figure 2a shows PC1, PC5, PC6 and PC7 resulted from the transformation. Figure $2 \mathrm{~b}$ are the same PCs but convolved with textural and directional filters, thus showing noise components more clearly.

Figure 3 is a graphical representa tion of the eigenvectors obtained from PC transformation on the raw subscene. Before visual analysis of the PC imagery, careful examination of the eigenmatrix can reveal some important information about the structure of the 
Table 1 - PC transformation on the seven reflective channels of the northern Eritrea JERS-1 subscene. Raw data; no stretch applied prior to the transformation

\begin{tabular}{llllllll} 
& \multicolumn{7}{c}{ STATISTICS } \\
DATA & OPS1 & OPS2 & OPS3 & OPS5 & OPS6 & OPS7 & OPS8 \\
MEAN & 52.44 & 62.03 & 49.58 & 71.40 & 44.30 & 55.70 & 42.57 \\
STDEV & 05.17 & 08.06 & 07.49 & 12.21 & 06.61 & 07.51 & 04.63
\end{tabular}

$\begin{array}{llllllll}\text { DATA } & \text { OPS1 } & \text { OPS2 } & \text { OPS3 } & \text { OPS5 } & \text { OPS6 } & \text { OPS7 } & \text { OPS8 } \\ \text { OPS1 } & \mathbf{2 6 . 7 1} & & & & & & \\ \text { OPS2 } & 38.00 & \mathbf{6 4 . 9 5} & & & & & \\ \text { OPS3\&4 } & 31.67 & 52.21 & \mathbf{5 6 . 0 5} & & & & \\ \text { OPS5 } & 40.46 & 76.08 & 66.16 & \mathbf{1 4 9 . 1 7} & & & \\ \text { OPS6 } & 14.63 & 29.75 & 22.88 & 66.56 & \mathbf{4 3 . 7 3} & & \\ \text { OPS7 } & 17.95 & 36.16 & 28.74 & 78.41 & 46.35 & \mathbf{5 6 . 4 6} & \\ \text { OPS8 } & 14.09 & 25.71 & 19.64 & 48.27 & 24.09 & 27.65 & \mathbf{2 1 . 4 1}\end{array}$

$s^{2}=418.48\left(\right.$ sum of variance $\left.s^{2}\right)$

PC EIGENMATRIX BASED ON COVARIANCE STATISTICS

\section{EIGENVECTOR MATRIX EIGENVALUES}

\begin{tabular}{llllllllll} 
& OPS1 & OPS2 & OPS3 & OPS5 & OPS6 & OPS7 & OPS8 & $\lambda$ & $\lambda(\%)$ \\
PC1 & +0.21 & +0.38 & +0.33 & +0.65 & +0.30 & +0.36 & +0.22 & 331.68 & $79.25 \%$ \\
PC2 & +0.37 & +0.46 & +0.50 & -0.20 & -0.40 & -0.42 & -0.11 & 55.01 & $13.14 \%$ \\
PC3 & +0.33 & +0.36 & -0.19 & -0.64 & +0.40 & +0.38 & +0.05 & 12.63 & $3.02 \%$ \\
PC4 & -0.24 & -0.31 & +0.71 & -0.22 & +0.21 & +0.30 & -0.39 & 9.02 & $2.15 \%$ \\
PC5 & -0.02 & -0.28 & +0.31 & -0.23 & +0.10 & -0.17 & +0.85 & 4.24 & $1.01 \%$ \\
PC6 & -0.05 & +0.00 & +0.01 & -0.07 & -0.73 & +0.65 & +0.20 & 3.19 & $0.76 \%$ \\
PC7 & +0.80 & -0.57 & -0.02 & +0.10 & -0.04 & +0.07 & -0.11 & 2.69 & $0.64 \%$ \\
\multicolumn{2}{l}{$\lambda=\mathbf{4 1 8 . 4 8 8}$ (sum of eigenvalues) } & & & & $\lambda \%=\mathbf{1 0 0}$ &
\end{tabular}

original data and the results of the transformation. It can be shown that although the sum of the variance of the original channels (diagonal line of the variance-covariance matrix of Table 1) is equal to the sum of the variance of the principal components (their eigenvalues), the variances are now very different for each new variable PC (Davis, 1973). Therefore, following one of the PCA's basic premises, it is evident that there is no loss or addition of information or noise during the process but just a reorganisation of the data set (Davis, 1973). Each eigenvalue can be used to assess the percentage of the original scene variance making up successive
PCs. In addition, it is possible to roughly approximate the contribution of each band to the individual PC images, by examining the eigenvectors for the original bands.

From Table 1 and Figure 3 it is apparent that the first row of eigenvectors making up the first $\mathrm{PC}$ are all positive. $\mathrm{PC} 1$ accounts for $79.25 \%$ of the total variance for the raw data PC transform. Overall brightness, or albedo, is responsible for the strong correlation between multispectral image channels and PCA has effectively mapped this into $\mathrm{PC} 1$. The statistical dimensionality of the data (related to sensor gain and offset as well as spectral differences and 
(a)

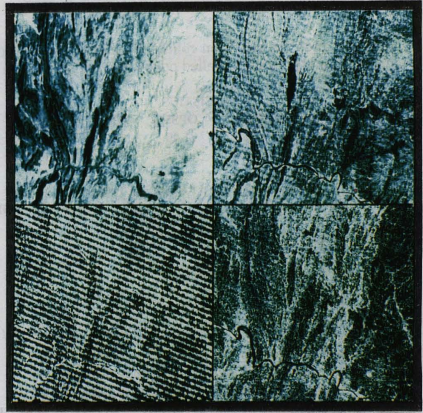

(b)

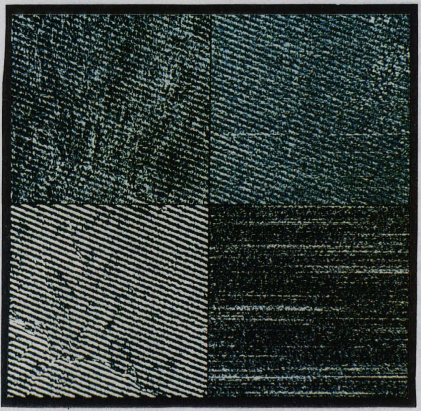

Figure 2 - (a) Examples of PCs calculated on the basis of all JERS-1 OPS channels of a selected subscene in Eritrea (Figure la). PCl, PC5, PC6 and PC7 correspond to the NW, NE, SW and SE quadrants, respectively. (b) Illustration of the same PCs of Figure $2 \mathrm{a}$, to which texture filters were applied to display the noise components evidently. PCl, PC5, PC6 and PC7 correspond to the same quadrants as in (a). 


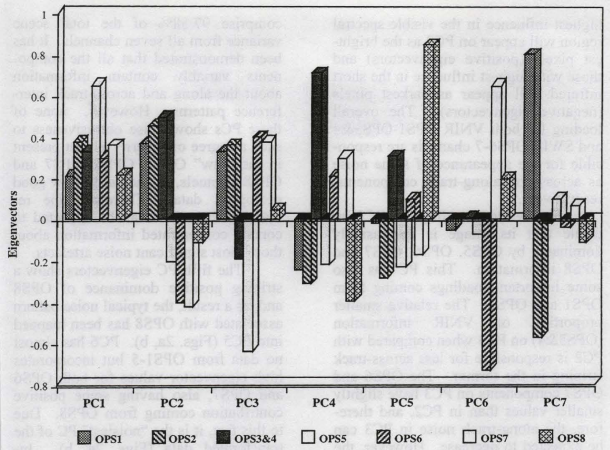

Figure 3 - Graphical representation of the eigenvectors from a $1024 \times 1024$ JERS-1 raw sub-scene of Eritrea

noise defects), and in particular, the magnitude of the standard deviations (SD), has great influence over the actual weighting of the original channels mapped into successive components. Examination of the magnitude and sign (i.e. positive or negative) of eigenvector loadings also gives an indication of which spectral properties of vegetation, rocks, soils and image defects are responsible for the statistical variance mapped into each $\mathrm{PC}$, and this is the basis for the noise isolation approach here examined.

In the raw data transformation of Table 1 the large SD for OPS5 (where noise is almost absent) is responsible for that channel's dominance in $\mathrm{PCl}$. Low SD of OPS1 (an important host of across-track noise) is largely responsible for OPS1's very low contribution to the same PC. This should mean that $\mathrm{PCl}$ has the higher signal-to-noise ratio (SNR) within the transform components. However, small contributions of OPS3 ('rich' in across-track striping) and OPS6 and OPS7 (the 'richest' in along-track noise) to $\mathrm{PC} 1$ will contribute some diffuse horizontal striping and diagonal patterns to this PC. This is confirmed in the enhanced $\mathrm{PCl}$ image of Figure $2 b$.

Eigenvectors loadings for $\mathrm{PC} 2$ of Table 1 indicate that it describes the difference between the visible channels (OPS1, OPS2 and OPS $3 \& 4$ ) and the SWIR channels (OPS5, OPS6, OPS7, OPS8). Image attributes which have 
highest influence in the visible spectral region will appear on $\mathrm{PC} 2$ as the brightest pixels (positive eigenvectors) and those with highest influence in the short infrared will appear as darkest pixels (negative eigenvectors). The overall loading for both VNIR OPS1-OPS $3 \& 4$ and SWIR OPS6-7 channels are responsible for the appearance of some noise as across and along-track components, respectively.

Eigenvectors loadings for PC3 indicate that its image is decreasingly dominated by OPS5, OPS6, OPS7 and OPS8 information. This PC has also some important loadings coming from OPS1 and OPS2. The relative smaller proportion of VNIR information (OPS3\&4) on PC3 when compared with $\mathrm{PC} 2$ is responsible for less across-track striping in the former. The OPS6 and OPS7 components on $\mathrm{PC} 3$ have slightly smaller values than in $\mathrm{PC} 2$, and therefore, the along-track noise in PC3 can be expected to decrease. However, the opposite relation is observed in the imagery (not shown) where PC3 shows a fine and tightly spaced subvertical striping. This 'noise enhancement' effect has been interpreted to be associated with the positive sign assigned to OPS6 and OPS7 eigenvectors loadings on $\mathrm{PC} 3$, as features associated with these channels will be shown as bright pixels.

Eigenvector loadings for PC4 (Fig. 3 ) suggest that it is dominated by bright OPS $3 \& 4$ scene attributes (positive eigenvector). Therefore, as a result, some obstructive horizontal striping can be expected in this PC. OPS1-OPS2 and OPS5-OPS8 contributions are either insignificant or represented by negative weights. The OPS6-OPS7 contribution to $\mathrm{PC} 4$ is lower than that of $\mathrm{PC} 3$, but again their positive eigenvectors are responsible for prominent appearance of some distinct artefacts.

So far, the first four PC images comprise $97.58 \%$ of the total scene variance from all seven channels. It has been demonstrated that all the components variably contain information about the along and across-track interference patterns. However, none of these PCs show noise obtrusiveness to such a degree of severity as that present in the "raw" OPS1, OPS6, OPS7 and OPS8 channels, but actually show good geological data. Therefore, the remaining three PCs can be expected to contain concentrated information about those most significant noise artefacts.

The fifth PC eigenvectors show a striking positive dominance of OPS 8 and, as a result, the typical noise pattern associated with OPS 8 has been mapped into PC5 (Figs. 2a, b). PC6 has almost no data from OPS $1-5$ but incorporates high eigenvector values for both OPS6 and OPS7, also having some positive contribution coming from OPS8. Due to this fact, it is the "noisiest" PC of the transformed data (Figs. 2a, b). Inversely, PC7 contains significant contribution from OPS1 and OPS2 whilst information from all other channels are irrelevant. As a consequence, horizontal striping is accumulated in this last PC (Figs. 2a, b).

The most important feature of this $\mathrm{PC}$ transformation is that the procedure was able to concentrate noise in the last two components, as anticipated. However, these two PCs contain information almost exclusively related to the very noisy OPS channels 1-2 and 6-7. Table 2 is a correlation matrix calculated on the basis of the variance-covariance matrix of Table 1 . Correlations between OPS 1 and 2 and OPS 6 and 7 are the highest within the matrix. The correlation matrix also shows that the VNIR channels have a high inter-correlation and a much lower correlation with the SWIR channels; and viceversa. These facts explain why the artefacts are concentrated independently in 
Table 2 - Correlation coefficients ( $r$ ) matrix derived from the covariance statistics displayed on Table 1.

$\begin{array}{lccccccc}\text { DATA } & \begin{array}{c}\text { OPS1 } \\ \text { OPS1 }\end{array} & \text { OPS2 } & \text { OPS3\&4 } & \text { OPS5 } & \text { OPS6 } & \text { OPS7 } & \text { OPS8 } \\ \text { OPS2 } & \underline{\mathbf{0 . 9 1 1 9}} & \mathbf{1 . 0 0 0 0} & & & & & \\ \text { OPS3\&4 } & 0.8178 & 0.8648 & \mathbf{1 . 0 0 0 0} & & & & \\ \text { OPS5 } & 0.6409 & 0.7730 & 0.7234 & \mathbf{1 . 0 0 0 0} & & & \\ \text { OPS6 } & 0.4281 & 0.5584 & 0.4621 & 0.8247 & \mathbf{1 . 0 0 0 0} & & \\ \text { OPS7 } & 0.4623 & 0.5973 & 0.5109 & 0.8550 & \underline{0.9337} & \mathbf{1 . 0 0 0 0} & \\ \text { OPS8 } & 0.5886 & 0.6889 & 0.5663 & 0.8538 & 0.7871 & 0.7951 & \mathbf{1 . 0 0 0 0}\end{array}$$$
\mathrm{r}_{\mathrm{i}, \mathrm{j}}=\mathrm{COV}_{\mathrm{i}, \mathrm{j}} / \mathrm{STD}_{\mathrm{i}} * \mathrm{STD}_{\mathrm{j}}
$$

the last two PCs.

\section{The Inverse Transformation}

The rationale of removing unwanted information in PC images by performing the inverse transformation is not new (Schowengerdt, 1983; Drury \& Hunt, 1988; Rothery \& Hunt, 1990; Hunt, 1991). Discarding noisy PCs before reverse transformation can remove some of the effects of interference patterns and consequently, improve the data's SNR. The unwanted PC data discard can be optionally achieved by either resetting all pixels of the unwanted PC (s) to zero or to a constant value; usually 127 (Rothery \& Hunt, 1990).

The forward transformation analysis highlights $\mathrm{PC} 6$ and $\mathrm{PC} 7$ as the noisy $\mathrm{PCs}$ to be cast off. Results obtained from using their images set at DN 127 or by completely omitting them from the inverse transform are fairly similar, although the former proved to retain a slightly better grey level gradient for the inverse transformed imagery.

The results from applying $\mathrm{PC}$ transformation to JERS-1 images showed several drawbacks. The forward PC transformation confined along across-track noise in the higher order PCs, but a significant amount of residual noise remained in the low-order components. Analysis of the variancecovariance matrix and correlation ma- trix extracted from the data indicates that this is probably because the noise defects, although similar in appearance, differ structurally from channel to channel, and hence, have a low degree of correlation in most bands. As a consequence, the transformation is unable to exclusively relegate the unwanted artefacts just to the higher order components. Ignoring the noisiest PCs before data decorrelation does improve the image quality after the data are converted back to wavebands. However, as previously stated, noise is still identified in all channels, indicating this technique to be partially useful, but unsatisfactory when compared with frequency domain methods.

Variants of the fundamental PCs transform, such as standardised PCs (Singh \& Harrison, 1985; Drury \& Hunt, 1988), selective PCs (Chavez, Jr \& Kwarteng, 1989), Gaussian-equalised raw channels (Loughlin, 1991), were also tested; the results on noise isolation all being basically the same. The noise removal methods based on the maximum noise fraction transform and minimum/maximum autocorrelation factors (Green et al., 1988), were not considered here; firstly, because the noise was cast to the last components by the standard PC transform; secondly, because the method proposed by Green et al. (1988), also involves filtering 
prior to the inverse transformation back to geographic space, offering no practi$\mathrm{cal}$ advantage. Another drawback to the PC-based methods applied to noise removal is their time and computational requirements; they necessarily involve forward and reverse transformations.

\section{FREQUENCY DOMAIN METH- ODS}

\section{Background}

An alternative approach to noise removal is to consider the noise components associated with images in the frequency domain. Images are transformed into the frequency domain using the Fast Fourier Transform (FFT). Once transformed into the frequency domain the magnitude component of the FFT can be used to help visualise the spatial frequency attributes of complex noise artefacts. Filters can then be applied in the frequency domain to remove noise artefacts before performing an Inverse Fourier Transform (IFT), to return the filtered image back to the spatial domain. The advantage of filtering in the frequency domain is that the operation allows the noise compo nent of an image to be easily identified and removed, without affecting any of the real image information. Such a task is always more difficult in the spatial domain.

\section{The Fourier Transform and its Com- ponents}

One of the simplest cases of image degradation is the occurrence of a sinusoidal interference structure superimposed on an image (Gonzalez \& Wintz, 1977). Such periodic function can be broken down into its most fundamental pieces, i.e. the sum of a series of superimposed trigonometric sine and cosine functions with different amplitudes, frequencies and phases. This representation of a function is termed its
Fourier series (Schowengerdt, 1983).

Figures $4 \mathrm{a}$ and $4 \mathrm{~b}$ show two sinusoidal functions of amplitude $A$, dependent just on a variable $X$, which can be expressed as:

Eq. 1

$f(X)=A * \cos (2 \pi X)$

Eq. 2

$$
f(X)=A * \cos (2 \pi 2 X) \text {, }
$$

respectively.

These correspond to low frequency (long wavelength) and high frequency (short wavelength) sine waves. The Fourier Transform of such continuous functions with variable $X$ can be defined by the equation:

$$
\begin{aligned}
& \text { Eq.3 } \\
& \qquad\{f(X)\}=F(U) \int_{-\infty}^{\pi} f(X) \exp [-2 \pi i U X] d X,
\end{aligned}
$$

where by Euler's formula exp $[-2 \pi i U X]$ $=\cos (2 \pi U X)-i \sin (2 \pi U X) ; i$ is the square root of -1 and $U$ is the so called frequency variable with respect to $X$.

An alternative way of representing $F(U)$ is in the form of its magnitude (or Fourier spectrum) $(|F(U)|)$ and phase angle $(\phi(U))$ components, that is:

Eq. 4

$F(U)=|F(U)| * e^{i \notin(U)}$, where

Eq. 5

$|F(U)|=\sqrt{R(U) * R(U)+I(U) * I(U)}$, and

Eq. 6

$f(U)=\tan ^{-1}\left[\frac{I(U)}{R(U)}\right]$,

$R(U)$ and $I(U)$ being the real and imaginary components of $F(U)$.

Digital images, however, are not continuous functions but discrete samplings of real scenes into image pixels. 
Hypothetical image analogues of these sinusoidal functions are shown in Figures $4 \mathrm{c}$ and $4 \mathrm{~d}$, whereas Figures $4 \mathrm{e}$ and 4f express these functions as lines of image data. In this case, still considering one dimension, it is assumed that there is a finite number of samples, $N$, and that the sampling is performed at regular intervals with a spacing of $x$. Thus the sample points are at $n x$, where $\mathrm{n}$ can have values of $0,1,2, \ldots, N-1$. The Fourier Transform of $N$ samples is defined to be,

\section{Eq. 7}

$$
F(U)=\frac{1}{N} \sum_{n=0}^{N-1} f(n x) \exp [-2 \pi i u n x]
$$

so that sinusoids in Eq.1 and Eq.2 can be re-written in the form:

\section{Eq. 8}

$f(n x)=A * \cos (2 \pi n)$,

and $A * \cos (2 \pi n)=A$ for $n=0,1, \ldots$

Eq. 9

$f(n x)=A * \cos (2 \pi 2 n)$,

and $A * \cos (2 \pi 2 n)=A$ for $n=0,1, \ldots$

However, Eq. 7 shows that $F(U)$ is still a continuous function, bringing problems for computing. To overcome this, in the same way that $f(X)$ is sampled, the transformed function can be sampled at $U=k u$, for $k=0,1,2, \ldots$, $N-1$, with $u=1 / N x$, that is,

Eq. 10

$F(k u)=\frac{1}{N} \sum_{n=0}^{N-1} f(n x) \exp [-2 \pi i k n / N]$

It can be shown by direct substitution of Eq. 8 into Eq. 10 that:

$$
\begin{aligned}
& F(k u)=0 \text { for } k \neq 1 \text { or } k \neq-1 \\
& F(k u)=A / 2 \text { for } k=1 \text { or } k=-1 \\
& F(k u)=A / 2
\end{aligned}
$$

$$
\begin{aligned}
& F(k u)=A \text { for } k=0 \\
& F(k u)=0 \text { for } k \neq 0
\end{aligned}
$$

and by direct substitution of Eq. 9 into Eq. 10 that:

$$
\begin{aligned}
& F(k u)=0 \text { for } k \neq 2 \text { or } k \neq-2 \\
& F(k u)=A / 2 \text { for } k=2 \text { or } k=-2 \\
& |F(k u)|=A / 2
\end{aligned}
$$

Figures $4 \mathrm{~g}$ and $4 \mathrm{~h}$ show schematic diagrams of the magnitude component for both cases. They look very similar, except that the spikes are positioned at higher values as the sinusoid assumes more cycles per given distance interval. Note that he FFT axes in the diagrams are the "wrong" way round when compared with the conventional arrangement (see Gonzalez \& Wintz, 1977; Schowengerdt, 1983), but this is an arbitrary convention. We use such "un usual" ordering because the bulk of our FFT code runs much faster in that way.

An image in the spatial domain represents variations in energy levels over geographic space whilst the Fourier transform of this image represents these energy levels with regard to variations of frequency. These frequency variations can be viewed either via as the plots of Figures $4 \mathrm{~g}$ and $4 \mathrm{~h}$, or more usefully in image form (intensity function), by looking at the intensity values in the magnitude plot produced by the FFT. Intensity or brightness in the magnitude plot is proportional to the amplitude of $|F(k u)|$. Figures $4 \mathrm{i}$ and $4 \mathrm{j}$ schematically represent such intensityfunction plots where the spots along the $k u$ axis consist of a single frequency in the input image in the $n x$ direction; the closer the spots to the origin the lower the frequency. Consequently the Fourier Transform of a sinusoid corresponding to a pair of spikes (or bright spots in a computer screen) will have a 


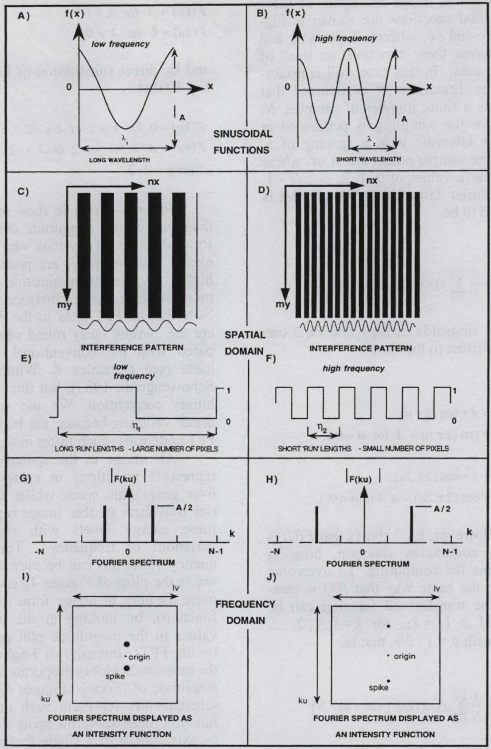

Figure 4 - Fundamentals of the frequency domain (see text for explanation). The graphics are only sketches and do not represent synthetic images of senoids. Also note that the axis of the images in the frequency domain are inverted in relation to those in the spatial domain. That is the standard that we follow in the paper. 
distance from the origin which is proportional to the frequency of the original sinusoidal function.

A magnitude plot, therefore, displays spatial frequency variations radi ally from a central origin of zero frequency (Fig. 5). High spatial frequencies or very fine gratings (i.e. short 'run' lengths, comprising a small number of image pixels) correspond to points furthest away from the origin, whereas low spatial frequencies or large gratings (i.e. long 'run' lengths, comprising a large number of image pixels) correspond to points closest to the origin (Fig. 5). The majority of the image detail occupies a broad zone, centred about the zero frequency point or origin (centre of Fig. 5). The shape and extent of this zone will vary on all magnitude plots as it depends directly on the distribution of spatial frequencies in the real image. For example, an image of a relatively homogeneous region with few edges (either spectral or topographic) would plot close to the magnitude origin at low frequencies, whereas a rapidly changing region with numerous edges would plot further away from the origin at higher frequencies. A circular shape to this central zone represents a random distribution of edge information in the original image. A strong preferred orientation of features in the original image data would cause this region to be elongated about the central origin.

A regular periodic structure in an image, such as noise (Figs. $4 \mathrm{a}$ and $4 \mathrm{~b}$ ) needs only a few gratings to represent it and will have a Fourier transform were all of the energy is constrained in a well defined bright spot (or spots) in the magnitude plot (Figs. $4 \mathrm{i}$ and $4 \mathrm{j}$ ). Figure 4a shows a schematic representation of a low frequency sinusoidal function superimposed on an image to produce along-track noise. In image terms this low frequency noise would comprise long 'run' lengths of image pixels repre-

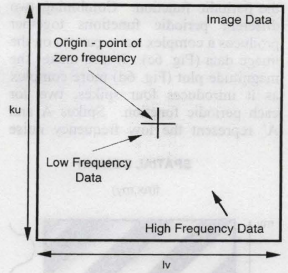

Figure 5 - Magnitude plot chracteristics.

senting noise in the spatial domain. Figure $4 \mathrm{i}$ shows that this periodic noise produces two spikes in the magnitude plot. These are located relatively close to the origin of the magnitude plot. A high frequency sinusoidal noise function (Fig. 4b) also produces along-track noise. Displayed as an image, such a function is represented by short run lengths of pixels. On the schematic magnitude plot (Fig. 4j) this noise is represented by two smaller spikes located further away from the image origin at higher frequencies. These spikes are "smaller", i.e. lower in intensity when compared to those of Figure 4i, because the amplitude of the original sinusoidal function is less than that of the low frequency noise in Figure 4a.

Introducing a periodic noise component in both the $\mathrm{x}$ and $\mathrm{y}$ directions simultaneously has the combined effect of producing a diagonal superimposed periodic noise structure at an angle ø to the image axis (Fig. 6a). This results in the noise spikes in the magnitude plot being rotated off axis by the equivalent angle (Fig. 6b). The distance of these spikes away from the origin is still determined by the original frequency of 
the periodic function. Combining two different periodic functions together produces a complex noise pattern on the image data (Fig. 6c). It also makes the magnitude plot (Fig. 6d) more complex as it introduces four spikes, two for each periodic function. Spikes $\mathrm{A}$ and $A^{\prime}$ represent the low frequency noise

\section{SPATIAL DOMAIN}

$f(n x, m y)$

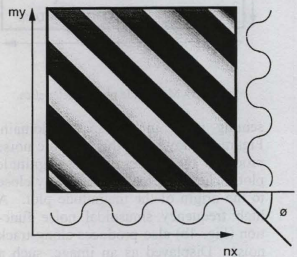

(a)

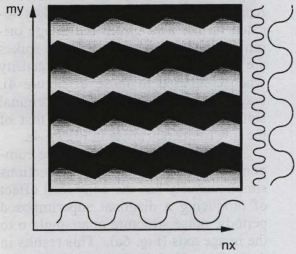

(c) equivalent to that of Figure $4 \mathrm{a}$ whilst spikes B and B' represent the slightly higher frequency noise equivalent to that of Figure 4b. Note that A and A' have a higher intensity than B and B' (schematic represented as larger spots) because of the higher amplitude of their original input function.

FREQUENCY DOMAIN

$\mathrm{F}(\mathrm{ku}, \mathrm{lv})$

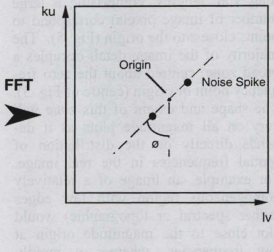

(b)

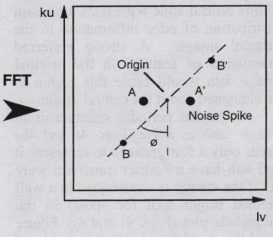

(d)

Figure 6 - Periodic complex noise (see text for explanation). The graphics are only sketches and do not represent synthetic images of senoids. Also note that the axis of the images in the frequency domain are inverted in relation to those in the spatial domain. That is the standard that we adopted in the paper. 
These simple schematic examples (Figs. 4-6) show how image noise can be regarded as the superimposition of different sinusoidal functions, with different amplitudes and frequencies, parallel to one or both image axes. Varying any one of these function components results in a very distinct noise structure. These examples also demonstrate how variations in the sinusoidal function are represented as variations in the magnitude plots. Considering these examples first helps when trying to understand the complex structures found in real noisy image data. Although, without a thorough knowledge of all of the processes which have affected corrupted image data from the time of acquisition, through pre-processing to final product distribution, it may be impossible to explain all of the features seen in a magnitude plot. However, the majority of noise artefacts associated with JERS-1 OPS, SPOT and AVHRR data produce features in the frequency domain, which can be recognised as being distinct from the image data.

\section{Periodic (Coherent) Noise Removal in The Frequency Domain}

As already mentioned the major advantage of transforming images into the frequency domain is that the periodic noise component of an image can be distinguished as features independent of the real image data. This means that processing in the frequency domain can be used to either suppress or totally remove systematic noise defects. Removal of noise is accomplished by filtering the magnitude plot before performing the Inverse Fourier Transformation (IFT) to return the data back to the spatial domain.

Although this may sound simple it is in fact quite a difficult task to perform. This is because the noise component can be masked or removed by numerous techniques and the suitability of any one technique will depend on the nature of the noise being removed. Care also has to be taken when processing in the frequency domain to ensure that minimal real image information is lost or removed when masking the noise component. Four approaches to noise removal in the frequency domain are discussed and demonstrated here.

\section{Notch Filters}

The simplest processing technique available to remove periodic noise from magnitude plots is that of Notch filtering. This technique relies on the insertion of a zero weighted mask over any feature in a magnitude plot which represents a noise artefact (Fig. 7). These masks should ideally be the exact size of the feature they are masking to ensure that no real image data is removed. This is quite easy to accomplish for noise of a consistent periodicity which produces a well defined high intensity spike in the magnitude plot. But it is not very easy to accomplish for features which do not have a consistent periodicity and therefore produce broad spikes in the magnitude plot. Masking these to zero may result in the loss of some image detail, which is contained within their broad 'skirts' (Gonzalez \&

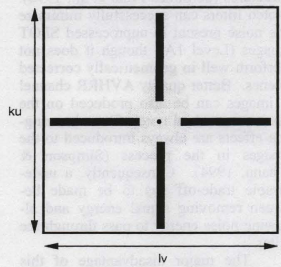

Figure 7 - Schematic Fourier notch filters 
Wintz, 1977). Problems also occur when trying to mask even a well defined spike which occurs at fairly low frequencies as it may be contained within the central zone of image detail. Even perfect delineation of such a point will result in the loss of image detail, as the spike will be partially comprised of real image detail as well as a noise component. One way of reducing the loss of image detail in such circumstances is to mask the point with a non zero value, a suitable value can be determined by interpolation from noise free regions from the surrounding magnitude plot (Schowengerdt, 1983).

Notch filtering is a technique which is best used to remove only well defined periodic features seen in a magnitude plot, preferably features that produce bursts of concentrated energy at a certain distance from the image origin. It is best applied to image data such as the JERS-1 Level-0 data, specifically the VNIR channels, which exhibit elementary noise structures in the frequency domain. Notch filters are capable of removing the across-track noise seen in the JERS-1 OPS VNIR channels, although their application to SWIR data is almost impossible; SWIR channels are corrupted by too many complex structures (de Souza Filho et al., 1996). Notch filters can successfully minimize the noise present in unprocessed SPOT images (Level 1A), though it does not perform well in geometrically corrected scenes. Better quality AVHRR channel 3 images can be also produced on the basis of standard notch filters but ringing effects are always introduced to the images in the process (Simpson \& Yhann, 1994). Consequently, a undesirable trade-off has to be made between removing signal energy and allowing noise energy to pass through the filter.

The major disadvantage of this process, despite of its limited success, is that of time. This process is extremely slow to implement as the magnitude plot for each channel has to be examined manually, and appropriate notch filters created. The process can be speeded up slightly by inserting the zero weighted notches into a constant image with a background DN value of one. This creates a binary mask which can be used to multiply any magnitude plot by, mapping 'notched' areas to zero whilst leaving the rest of the image unaffected. A problem associated with Notch filtering is that sharp edges in the filter may introduce spurious unwanted patterns in the spatial domain, such as ringing (Gonzalez \& Wintz, 1977).

Low-Pass Circular and Elliptically Symmetrical Fourier Filters

Another approach to noise removal is to use a low-pass filter by combining a magnitude plot multiplicatively with a large filter centred at low frequencies. These filters are usually circular or elliptical in shape and are weighted from one DN at their centre to zero $\mathrm{DN}$ at their edge. This weighting can either be a straight forward linear slope or it can be controlled by a predetermined function, such as a gaussian or logarithmic profile. These filters are located so that during multiplication their centres correspond with the zero frequency origin of the magnitude plot (Fig. 8). These filters have the affect of removing the high frequency component of the magnitude plot whilst preserving the lower frequencies. The extent of the region preserved depends both on the shape of the filter and on the weighting profile. This technique tends to eliminate the majority of high frequency noise structures although any noise at low frequencies tends to be preserved along with the image detail. Vice versa, any high frequency image detail, such as edge information, is lost along with the noise component. This results in the filtered images, once transformed 


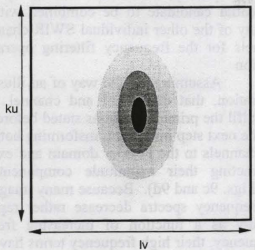

Figure 8 - Schematic low-pass elliptically simmetrical Fourier filter.

back into the spatial domain having a slightly blurred appearance, typically found in spatial domain low-pass filtering.

The advantage of this technique is that it is very quick to implement compared to that of Notch filtering. The problem lies in the fact that this is a very basic technique which removes artefacts at the expense of real image data. Tests of this technique show that it works reasonably well on both Level0 and Level-2 VNIR data, as it is capable of removing across-track noise. However, problems arise with the SWIR channels as these (especially Level-2 data) contain significant noise structures at low frequencies. Removal of these features using this technique means that a very small filter has to be used, which results in only minimal image detail being preserved. This produces a cleaned output image with very minimal textural information preserved. Similar or even worse results are produced by applying such filters to SPOT and AVHRR channel 3 data.

A variation of this technique is to combine these low-pass filters with isolated notch filters. This combined technique means that the low-pass filter can be large, which helps to preserve the maximum amount of high frequency textural information, whilst isolated notch filters ensure that specific high or low frequency noise spikes are removed. The problem with this approach is once again the time factor, as ideally individual filters need to be produced for each image channel. Tests of this combined approach have produced some favourable results for both Level0 and Level-2 OPS data noise removal.

\section{Zonal Notch Filter (ZNF)}

The use of both standard notch and symmetrical low-pass filters, as described above can solve some noise problems within the image data considered in this paper. This is because the noise that is within these channels does not coincide with the range of frequencies representing the majority of the image data. There are situations, however, when important noise components fall within the frequency range of data representing the real scene. In other words, the noise energy is mixed with the image energy at the low frequency end of the range. This typically occurs in OPS SWIR channels 6,7 and 8 (Figs. $9 \mathrm{a}$ and $9 \mathrm{c}$ ), in SPOT panchromatic scenes (Figs. 14a and 14b) and AVHRR channel 3 data (Figs. 15a and 15c), which are severely affected by a multitude of interference patterns, represented by numerous aligned bright spots, varying from high to low frequencies. Experiments over these images using very small elliptical low-pass filters show that noise can be reduced, but at the expense of leaving out important high frequency image information. This causes the inverted FFT image to look blurred and devoid of useful textural and edge information. Because of these noise characteristics, the approaches that Rose (1989) and Hummer-Miller (1990) used to remove the noise in AVIRIS and TIMS images, respectively, caused by a 
few discrete sinusoidal harmonics at frequencies where there is little image signal energy, are not suitable for use.

The problem faced with the data employed here is "how one can isolate the noise energy from the signal energy, along high to low-frequencies, without mixing their information ?". Figures $9 \mathrm{c}, 14 \mathrm{~b}$ and $15 \mathrm{c}$ show that the DNs comprising noise and most signal information are alike, making their discrimination impossible (e.g., by histogram manipulation).

A pair of channels with highly correlated noise structures (at similar frequencies), but with poorly correlated scene information could provide the means to isolate the signal from the noise present in any multichannel data. It follows from this that a very accurate filter, which is scene dependent, can be derived from the difference between these two channels in the frequency domain. From this hypothesis, de Souza Filho et al. (1996) found that the above condition can usually be satisfied by at least two of the JERS-1 channels. Stemming from that, they developed a method to restore JERS-1 images quite successfully using Fourier operators.

The choice of a pair of channels covering the specifications mentioned above, strongly depends upon the individual scene information. However, some general rules can be addressed. It is demonstrable that the spectral information present in JERS-1 OPS channels $1,2,3$ and 5 data is variable, but is distinct from that contained in channels 6 , 7 and 8. This means that scene information between these sets are likely to be poorly correlated. The noise components are much more obtrusive in channels 6,7 and 8 than in any of the other OPS channels. The OPS VNIR channels are the least corrupted. Channel 5 is the least affected by additive noise within the SWIR set, but always contains it (Figs. $9 \mathrm{~b}$ and $9 \mathrm{~d}$ ). Channel 5 , consequently, will always rank as a potential candidate to be combined with any of the other individual SWIR channels for the frequency filtering operation.

Assuming, by the way of an illustration, that channel 5 and channel 8 fulfil the prime conditions stated before, the next step involves transforming both channels to the Fourier domain and extracting their magnitude components (Figs. $9 \mathrm{c}$ and 9d). Because many image frequency spectra decrease rather rapidly as a function of increasing frequency, their high frequency terms have a tendency to become obscured when displayed in image form (Gonzalez \& Wintz, 1977). We applied a gaussian contrast stretch to the magnitude plots to bring-out this low-level information, which, if shown simply as a standard magnitude plot, is beyond the dynamic range of the display system. The visual result of such contrast-stretch is similar to that of applying the traditional logarithmic re-scaling to the magnitude plot (Gonzalez \& Wintz, 1977), but has the advantage of being a data independent, contrast enhancing technique.

Considering that in both magnitude images: (i) the same gaussianstretch parameters are applied, (ii) the Cartesian position of the worst defects (spots and spikes) approximately coincide, and (iii) there is a reasonable difference between the scene information, then, the majority of the noise components should be detected and isolated by subtracting the magnitude plot of the channel that shows the noise structures most predominantly from the other. Figure $9 \mathrm{e}$ shows the magnitude plot resulting from the difference between the magnitude plots of channel 8 (Fig. 9c) and channel 5 (Fig. 9d). It is evident from Figure 9c that there is no apparent separability between the grey levels representing the periodic noise structures from those representing the rest of the 
image data (e.g. the DN values for the centre of the spikes have close values to those at and around the zero frequency point). The difference between the magnitude images (DMI) (Fig. 9e), however, shows all the noise components to be concentrated at low DN values (dark pixels), while the pixels comprising the rest of the image signal remain at much higher DN levels (brighter pixels). The resulting DMI itself, although distinguishing the noise, would be difficult to interpret if transformed back to the spatial domain. However, it can be used as a basis for creating an effective scene-dependent filter. Such a filter can be produced, firstly, by re-scaling the DMI, produced by the above procedure to form a byte image. A threshold value is estimated which separates the noise component of the image from that of the real image component more precisely. This threshold is best found using an interactive pseudo-colouring routine to highlight the values representing noise. Once a threshold value which represents the break point between the noise and the real image components is found, a simple linear mapping function can be applied. This function is used to map all of the values in the DMI representing noise to zero, and to map all of the real image data to one, thereby creating a binary mask. Figure $9 f$ illustrates a binary mask created by this procedure (the DN values for noise and image signals were mapped to 255 and 0 , respectively, for greater clarity). Such a mask is termed the 'Zonal Notch Filter' (ZNF) (de Souza Filho et al., 1996). Note that all noise components are isolated in the process, including some other minor noise represented by small bright spots away from the main 'noise axes'. Figure 10 outlines the processing steps involved in creating the $\mathrm{ZNF}$.

Once a $\mathrm{ZNF}$ has been created it is combined multiplicatively with each channel's FFT. This removes the unwanted noise component of the original image, as this is mapped to zero in the frequency domain. Figure 11 shows the processing steps for using this filter to remove noise from an original input image. ZNFs will remove all noise components introduced by both the optical sensor failures and those caused by the pre-processing algorithms, without removing too much of the image information, providing that a suitable break point is chosen. Because the $\mathrm{ZNF}$ is a bit image, and because the spatial frequency of the noise is unaffected by stretched or unstretched magnitude plots, the multiplication of a ZNF to either, produces identical results as regards noise reduction after the images are transformed back to the spatial domain. However, the use of gaussianstretched magnitude brings some special effects to the scene in the spatial domain, which can be exploited to minimise brightness saturation problems within JERS-1, SPOT and AVHRR data (de Souza Filho et al., 1996). A gaussian stretch applied to the magnitude plot can re-order the spatial frequencies into a gaussian distribution, yielding images that shows balanced luminance gradients, increased dynamic range and more uniform display of local details.

Figures $9 \mathrm{~g}$ and $9 \mathrm{~h}$ show ZNF-re stored JERS-1 OPS channel 8 and JERS-1 OPS channel 6 images. The original images (Figs. 1a-9a and 1b) contain large amounts of both horizontal and sub-vertical noise structures in the spatial domain, which represent a variety of complex interference patterns in the frequency domain. Applying the $\mathrm{ZNF}$ removed all of these interference patterns as well as the brightness saturation, and has yielded images showing a significant improvement in quality. All OPS channels filtered by these methods exhibit similar improvements, 

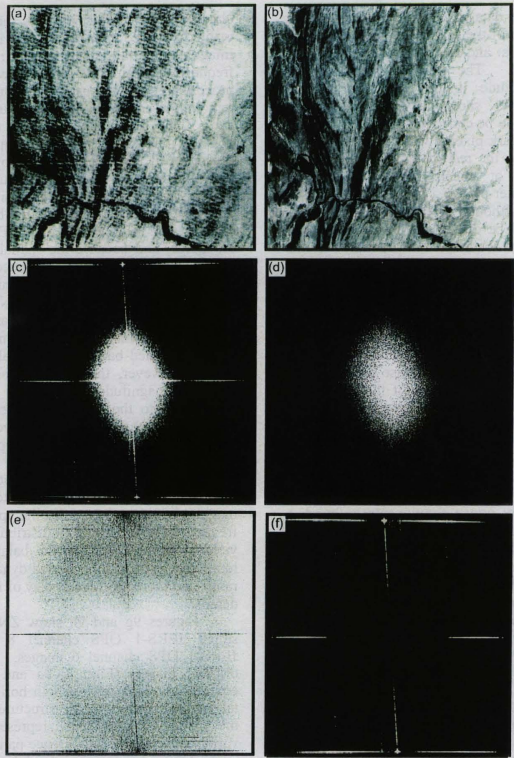

Figure 9 - (a) Level-2 raw JERS-1 OPS channel 8 data; (b) Level-2 raw JERS-1 OPS channel 5 data; (c) Magnitude plot derived from the JERS-1 OPS channel 8 of Figure (a); (d) Magnitude plot derived from JERS-1 OPS channel 5 of Figure (b); (e) difference magnitude image (DMI), resulted from subtracting the magnitude plots of channels 5 and 8; (f) Zonal Notch Filter (ZNF) derived from this DMI (in this picture, all of the values representing the estimated noise were mapped to 255 and the rest of the image to zero, for greater clarity); 

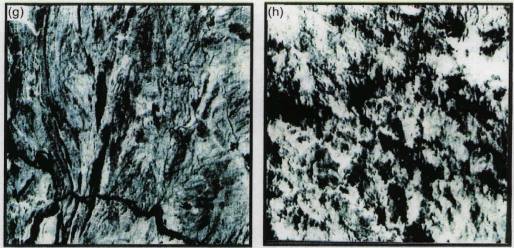

Figure 9 (conclusion) - (g) Restored Level 2 JERS-1 SWIR OPS channel 8 data (compare with Fig. 1a); (h) Restored Level 2 JERS-1 SWIR OPS channel 6 data (compare with Fig. 1b).

although achievements over SWIR channels 6 and 7 may vary according to scene information and original data quality.

\section{DISCUSSION}

Among the noise minimisation techniques experimented in this paper, the PC transformation is clearly the least effective. The PC method is not able to completely isolate the noise from the image signal. Even the PCs containing very high signal-to-noise ratio (SNR), such as PC1, show considerable amount of remaining features related to along- and across-track noise.

The other restoration methods, however, show closer visual results which can not be assessed solely in the spatial domain (Figs. 9g and 9h). In this case, analysis of the Fourier spectra of previously filtered images is a fine way to evaluate how effective a filtering technique can be. Figure 12a shows the magnitude plot (half image) of the OPS channel 8 (Fig. 1a; 9a) filtered via a spatial domain low pass filter (Kernel 6). The result of applying efficient elliptically symmetrical Fourier filters to the same image is practically identi- cal and therefore not shown. In both cases, noise is eliminated or reduced from high-to-mid frequencies, but there is still a significant number of star-like components representing noise towards mid-to-low frequencies. The remaining noise is not completely obtrusive in the spatial domain, seeming to be diluted within the image signal. However, it can be greatly enhanced if a texture filter is applied (lower half of Fig. 12a). Another considerable drawback of these methods is that of while eliminating high frequency noise they also tend to eliminate high frequency image signal, producing undesirable levels of blur and/or images lacking in topographic expression.

Figure $12 \mathrm{~b}$ represents the magnitude plot of the same image but restored by the frequency domain Zonal Notch Filter (ZNF) technique. It shows the high degree of efficiency achieved by the method when compared with those previously discussed. Basically, no noise structures are left in the image and most of the signal impulses, from lowto-high frequencies, are preserved. This method is the optimal procedure to reduce problems in multichannel data. A drawback of this technique, however, is 


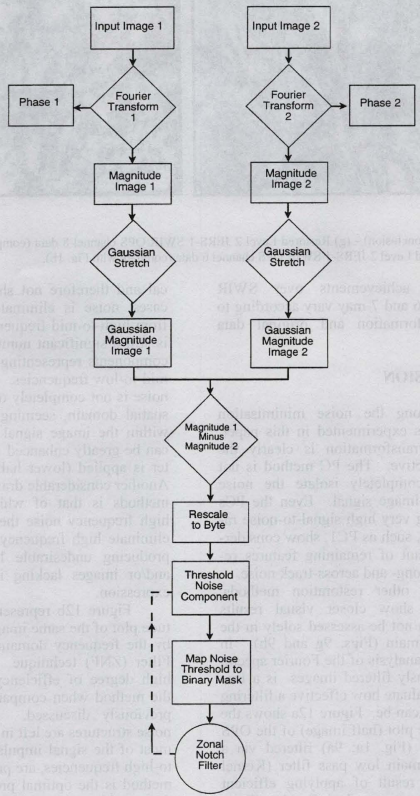

Figure 10 - Flow diagram for producing a Zonal Notch Filter from two input channels. 


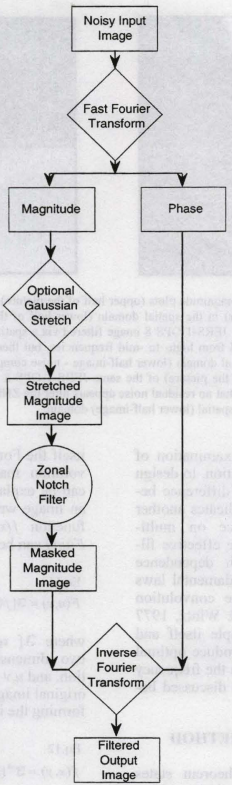

Figure 11 - Flow diagram of the steps required for a complete restoration (noise removal and brightness equalization) of a single input channel. 
(a)

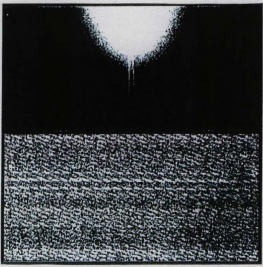

(b)

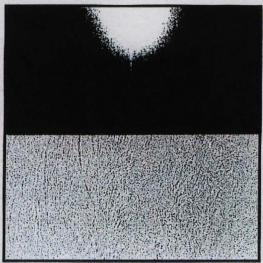

Figure 12 - Digital mosaic of magnitude plots (upper half of the picture) and noise-enhanced images (i.e., convolved with a texture filter) in the spatial domain (lower half of the picture). ((a) Magnitude plot (upper half of the picture) of a JERS-1 OPS 8 image filtered via a spatial domain low pass filter (Kernel 6). Note that noise is reduced from high- to -mid frequencies, but there is still a significant amount of remaining artefacts in the spatial domain (lower half-image - noise component in the spatial domain); (b) Magnitude plot (upper half of the picture) of the same JERS-1 OPS 8 but restored by the Zonal Notch Filter (ZNF) technique. Note that no residual noise appears after the ZNF is applied both in the frequency (upper half of the picture) and spatial (lower half-image) domain.

that it requires careful examination of interchannel noise correlation, to design filtering masks from the difference between channels. This indicates another problem; the dependence on multichannel analysis to create effective filters. Overcoming such dependence could use one of the fundamental laws in image restoration: the convolution theorem (see Gonzalez \& Wintz, 1977 for details). The principle itself and other possible ways to produce optimal filters based on it (both in the frequency and spatial domains), are discussed below.

\section{THE SYNERGISTIC METHOD}

The convolution theorem states that the operation of convolution with a kernel in the spatial domain is equivalent to multiplication in the frequency (Fourier) domain by a function which is itself the Fourier Transform of the convolution mask. Mathematically, this can be explained as follows. Consider an image written as a two dimensional function $f(x, y)$; its Fourier transform $F(u, v)$ can be written as:

$$
\begin{aligned}
& \text { Eq.11 } \\
& F(u, v)=\Im(f(x, y)
\end{aligned}
$$

where $\mathfrak{I}\{$ represents the operation of two dimensional Fourier transformation, and $u, v$ are spatial frequencies. The original image may be recovered by performing the inverse transformation, i.e.

\section{Eq.12}

$f(x, y)=\mathfrak{J}^{-1}\{F\{u, v)$

Now, suppose that image $f(x, y)$ is convolved with a kernel $k(x, y)$ in the spatial domain to form a new image 
$f_{\text {conv }}(x, y)$, i.e.:

Eq.13

$f_{\text {conv }}(x, y)=f(x, y) \times k(x, y)$

where * represents the operation convolution. The kernel $k(x, y)$ obviously modifies the relationship between the various spatial frequencies comprising the image $f(x, y)$. It is as if the Fourier transform had been multiplied by a spatial frequency weighting function or spatial frequency filter. Mathemati- cally, this can be written as,

\section{Eq.14}

$F_{\text {canv }}(u, v)=F(u, v) * K(u, v)$

where $F_{\text {conv }}$ and $F$ are the Fourier transforms of $f_{\text {conv }}$ and $f$ respectively, and $K$ is the spatial frequency filtering function. The most important relation for practical purposes is the following,

\section{Eq. 15}

$K(u, v)=\Im\{k(x, y)\}$

\section{Example 1}

\section{FREQUENCY FILTER}

\section{Zonal Notch Filter}

- ideal frequency domain mask
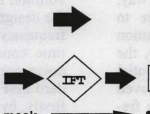

- $1024 \times 1024$ pixels Fourier filter

\section{SPATIAL FILTER}

\section{Convolution Kernel}

- ideal spatial domain mask

- $1024 \times 1024$ pixels convolution filter

\section{Example 2}

\section{SPATIAL FILTER}

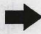

\section{FREQUENCY FILTER}
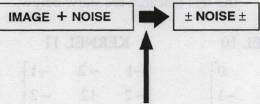

$\left[\begin{array}{lll}-1 & -2 & -1\end{array}\right]$

$\left|\begin{array}{lll}-2 & 12 & -2\end{array}\right|$

$\left\lfloor\begin{array}{lll}-1 & -2 & -1\end{array}\right]$

High Pass Convolution Filter

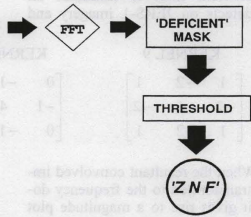

Figure 13 - Applications of the synergistic method for filter design. 
From Eq. 15, it can be said that the operation of several convolution kernels in sequence to the same image simply results in the Fourier transform of the image being multiplied by a succession of spatial filter functions. If one form the product of all these filters, and then apply the composite filter to the image's Fourier transform in one go, the result is identical. One could also find the corresponding convolution kernel by applying Eq. 15 in reverse. This convolution kernel can be obtained by forming the convolution of all the individual kernels in sequence (this can be proven using Eq. 14).

Eq.15, therefore, can be extremely useful as it unfolds a two-way system for filter design (Fig. 13). In one way, based on its reverse, a procedure to choose the elements of a convolution kernel can be established. Firstly, the operator chooses the range of spatial frequencies which need to be removed in the frequency domain (e.g. high frequency for noise), and constructs a Fourier function which weights these frequencies accordingly. Having arrived at a filter, the next step consists of performing the inverse Fourier transform on the function to meet the desired convolution kernel. The Zonal Notch Filter previously described proved to be the most efficient filter to minimise all noise defects on JERS-1 imagery and therefore, the inverse Fourier Transform of such 'function' gives rise to an optimal convolution kernel in the spatial domain. This kernel obviously will have the same size as the image (i.e. $1024 \times 1024$ elements), but many of the elements not near the centre may be quite small, and are often substituted by zeros to reduce the matrix size. Experiments with convolution kernels produced in such a way were also explored to some extent during this research. The results are very similar to those obtained by the use of their parental Fourier filters and will not be repeated here.

Perhaps more important, Eq. 15 also allows standard $3 \times 3$ spatial convolution filters to be used as a basis for the design of very efficient filters in the frequency domain, not requiring the time consuming analysis of interband noise correlation needed for the standard ZNF approach. This can be done, firstly, by convolving the noisy channel with an ordinary high pass convolution filter, aiming to separate the majority of the noise content from the image data. Small filters such as this, as said before, will perform a crude filtering with limited control on spatial frequencies; however, they have the advantage of requiring minimum computing time. Some useful high-pass filters used during this research are show below,
KERNEL 9

$$
\left[\begin{array}{rrr}
1 & -2 & 1 \\
-2 & 4 & -2 \\
1 & -2 & 1
\end{array}\right]
$$

When the resultant convolved image is transformed to the frequency domain, it gives rise to a magnitude plot containing all the typical regular pattern of spikes representing noise. Such magnitude plot will also show some in
KERNEL 10

$$
\left[\begin{array}{lrr}
0 & -1 & 0 \\
-1 & 4 & -1 \\
0 & -1 & 0
\end{array}\right]
$$

KERNEL 11

$$
\left[\begin{array}{lll}
-1 & -2 & -1 \\
-2 & 12 & -2 \\
-1 & -2 & -1
\end{array}\right]
$$

formation about the signal. This 'misbehaviour' of the filter can be corrected if a threshold value which separates the noise and the signal is determined. This can be achieved following the same steps described for the Zonal Notch 
Filter, both noise suppression techniques being performed identically from this stage. The results are obviously equivalent. The idea of this alternative method perhaps is not as elegant as that of the ZNF technique, but it brings the advantage of employing just one single channel to build up the same consistent Fourier filter.

The panchromatic SPOT image displayed in Figure $14 \mathrm{a}$ is amongst the data used here to demonstrate the efficiency of this method. Note in the magnitude plot of Figure 14b (derived from the image of Fig. 14a) that the noise components are rotated anticlockwise. That is because a geometric correction was performed in the original scene. This pre-processing further complicates the noise structure. After the resampling is applied, the noise is no longer coherent as it appears in uncorrected Level 1A data (Westin, 1990). That makes the isolation of noise a much more difficult task and therefore it is an excellent case to be used as a methodological proving ground. Figure $14 \mathrm{c}$ is a magnitude plot created from high pass convolution filtering of the geometrically corrected SPOT scene. Note that this image resembles the difference magnitude image created by the standard ZNF method (Fig. 9e). Here, the noise and image signal were separated to some degree into very low and intermediate- to -high DNs. By carefully examining the histogram of Figure $14 \mathrm{c}$, to determine a threshold value which separates the noise component from that of the real image data, one can produce the mask as displayed in Figure $14 \mathrm{~d}$. Observe that it is possible to modulate spatial frequencies representing only noise with extreme detail; virtually no image signal is mistaken by noise. Using this mask as a Fourier operator, following the procedures described in the last steps of the ZNF method (including the brightness satu- ration correction), yields a noise-free, high quality SPOT image as pictured in Figure 14e.

Figures $15 \mathrm{a}$ and $15 \mathrm{~b}$ are NOAA9 AVHRR channel 3 and channel 4 , respectively. The main noise associated with channel 3 is a complex of aliasing of high frequency noise (Simpson \& Yhann, 1994). The aliased noise produces the horizontal bands which run from the left to the right side of the magnitude plot of Figure $15 \mathrm{c}$. Figure $15 \mathrm{~d}$ is the magnitude plot of AVHRR channel 4; "a good channel 3" should show a magnitude plot similar to this. Figure $15 \mathrm{e}$ is the difference magnitude image (DMI), resulted from subtracting the magnitude plots of AVHRR channels 3 and 4 . Similarly as observed in the JERS-1 data, this technique isolates the noise from the image signal quite efficiently. Figure $15 \mathrm{f}$ is a magnitude derived from a high pass filter operation on the raw noisy AVHRR channel image. The results from both approaches are alike, though the latter does not require a second channel to modulate the noise. Figure $15 \mathrm{~g}$ and $15 \mathrm{~h}$ are Zonal Notch Filters produced with broad-band and narrower-band thresholds, respectively. Figures $15 \mathrm{i}$ and $15 \mathrm{j}$ are the corresponding restored images. Note that in this case both results are not so satisfactory. The resultant images are either too blurred and lacking textural information (Fig. 15i) or too noisy for adequate use (Figure 15j). The reason for this is because there are further complications in the imagery, apart from the noise isolated in process, that simply denied some more obscure noise components to be unmixed from the image signal. Although the method did not perform convincingly for this particular AVHRR data set, it is usually capable of producing good results. The AVRHH data presented here contain some of the worst defects ever to be found in images acquired by the NOAA 

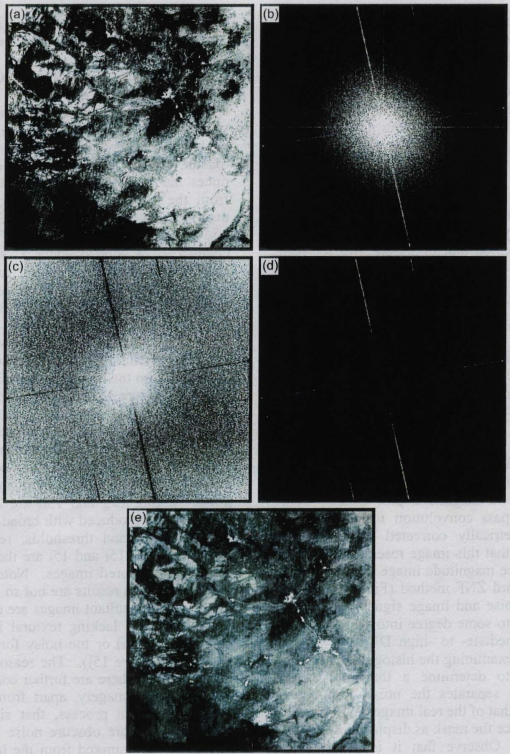

Figure 14 - (a) Geometrically corrected panchromatic SPOT 3 HVRI Level IA data; (b) Magnitude plot derived from the panchromatic SPOT 3 HVR1 Level IA data of Figure (a); (c) Magnitude plot created from high pass convolution filtering of SPOT 3 HVR1 Level 1A in the spatial domain; (d) Zonal Notch Filter (ZNF); (e) Restored panchromatic SPOT 3 HVRI Level IA data (compare with Figure Ic). 

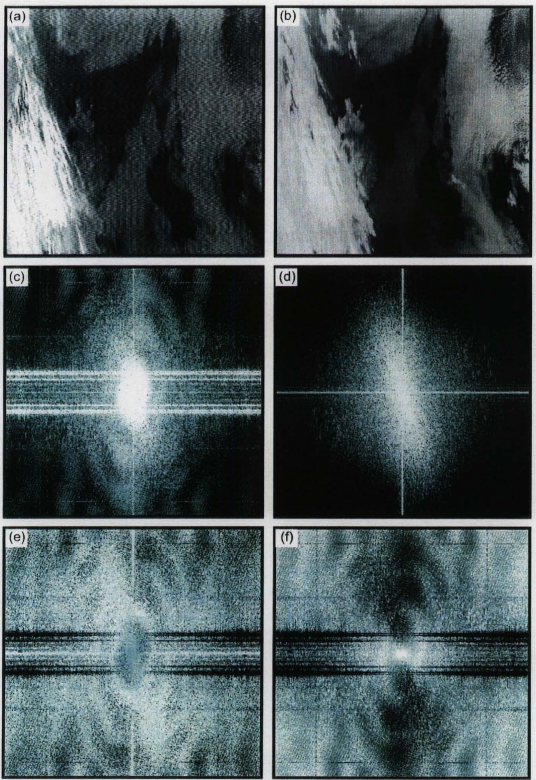

Figure 15 - (a) NOAA9 AVHRR channel 3 data; (b) NOAA9 AVHRR channel 4 data; (c) Magnitude plot derived from NOAA9 AVHRR channel 3; (d) Magnitude plot derived from NOAA9 AVHRR channel 4; (e) difference magnitude image (DMI), resulted from subtracting the magnitude plots of AVHRR channels 3 and 4; (f) Magnitude plot created from high pass convolution filtering of AVHRR channel 3 in the spatial domain. 


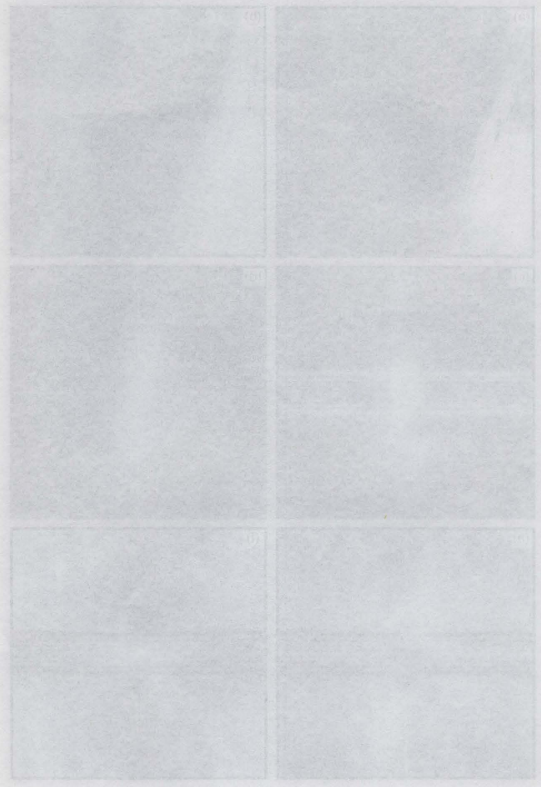

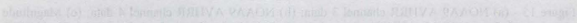

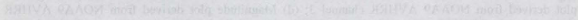

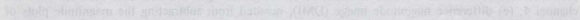

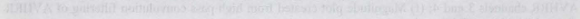

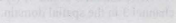



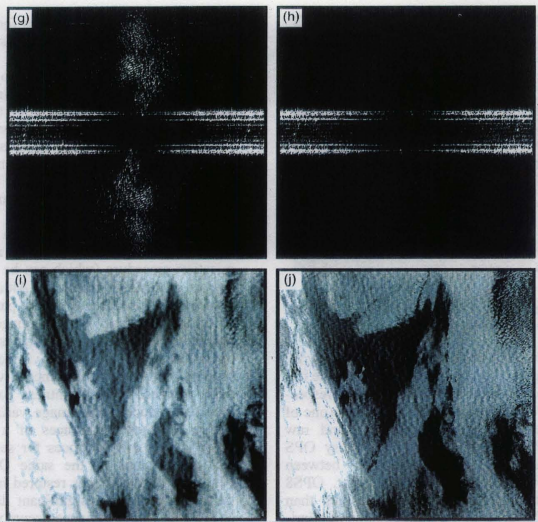

Figure 15 (conclusion) - (g) broad-band Zonal Notch Filter (ZNF); (h) narrow-band Zonal Notch Filter (ZNF); (i) Restored AVHRR channel 3 using a broad-band Zonal Notch Filter (ZNF); note that the image noise-free but at the expense of unaccetable levels of blurring; (j) Restored AVHRR channel 3 using a narrow-band Zonal Notch Filter (ZNF) (compare with Figs. 1d; 11 a and 1lb).

series.

\section{CONCLUSIONS AND WIDER IM- PLICATIONS}

Images restored by any of the methods described in this paper, despite their good quality and usefulness in many applications, are not recommended for use in quantitative measures of reflectance. The reasons for that vary from technique to technique and with the extent to which they are applied. Although the raw image hues can be well preserved in the whole process, relative brightness components can be modified, compromising any quantitative usage. The diagrams in Figure 17 show this,

Figure 16 is an image of the JERS-1 OPS8 estimated noise in the spatial domain, yielded from subtracting a restored image from the original image. Figure 17a illustrates the variation of the noise components in the spatial domain for 1024 pixels along an arbitrary row of Figure 16, while Figure $17 \mathrm{~b}$ shows the pattern of the noise for 1024 


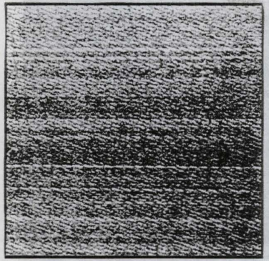

Figure 16 - JERS-1 OPS channel 8 estimated noise in the spatial domain.

down an arbitrary column of Figure 16. Figure $17 \mathrm{c}-\mathrm{d}$ and $17 \mathrm{e}-\mathrm{f}$ are the same image rows and columns extracted respectively, from raw OPS channel 8 (one of the noisiest OPS channels) and raw OPS channel 5 (the least noisy OPS channel). The difference between OPS5 and OPS8 data is clear, OPS8 showing far more noise spikes than OPS5. Ideally, a filter capable of eliminating the noise whilst retaining most of the image data would be the one able to preserve the gross appearance of the profile shown by OPS 5 , but free of those sharp spikes presented by OPS8. Figure $17 \mathrm{~g}-\mathrm{h}$ are the corresponding row and column extracted from the OPS8 restored by the ZNF technique, with no brightness correction applied in the frequency domain. The restored image is now comparable to OPS5 (Figure 17ef); i.e. it is smoother and it lacks in prominent periodic spikes. Some local modification has occurred, and postfiltering quantification may produce dubious results. The same problem affects images restored by any of the other techniques.

In cases where quantitative analysis is crucial, it should precede 'clean- up' filtering. The same applies for segmentation, enhancement and classification procedures. For example, techniques such as principal components analysis (PCA), decorrelation-stretch, ratio and intensity-saturation-hue (ISH) transformation, work better if clean-up filtering is performed on the final enhanced/classified images. The noise, which is greatly emphasised by these methods, is just as effectively removed post hoc.

Care is also needed when performing the brightness saturation minimisation procedure (which can be used as part of the ZNF method). Although it successfully reduces the large patches of saturated areas in the image by equalising low spatial frequencies, the operation may 'wash out' important spectral and tonal differences within these patches. The brightness correction also reduces total image variance. Figure 18 shows variances of a raw OPS 8 image and variances for several restored versions of the same OPS8. Note that for all images restored in the frequency domain, a significant difference on the image variance occurs when the brightness correction is applied. The variance in these cases are much lower. That is simply because there is a decrease in image saturation at the expense of illumination, the component responsible for the overall dynamic range of the scene. Despite these problems, there are situations when brightness saturation can completely avoid the concomitant observation of very bright and very dark areas in the imagery, so that it must be corrected. In this cases, the best way to avoid arbitrarily 'loss' of information is to inspect the tonal and spectral differences in the image prior to restoration.

Analysis of the total image variances on Figure 18 is also interesting as it confirms some previous results. The OPS8 restored by the ZNF method (no 


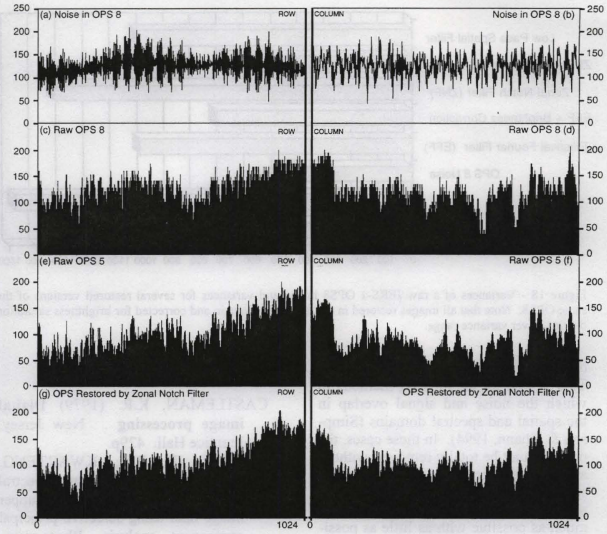

Figure 17 - Illustrations of the actual impact of the noise components over OPS images and their suppression via the Zonal Notch Filter technique. (a) (b) Variation of the noise components in the spatial domain for 1024 pixels along an arbitrary row and column in OPS8 (Figure 2C.4); (c) (d) Superposition of noise and image signal in the raw OPS 8 (Figure 2A.2b); (e) (f) Superposition of noise and image signal in the raw OPS 5 - note the absence of equidistant spikes when compared with plots in (c) and (d); (g) (h) OPS 8 restored via the Zonal Notch Filter technique - note the similarity between these plots and those of $(\mathrm{e})$ and (f). All images were scaled to the same $\mathrm{DN}$ range to avoid distortions.

brightness correction applied) contain the highest variance. This variance value is the closest to OPS channel 5 amongst all others, indicating the powerfulness of the technique to relegate noise, preserving the important image signal. The similarities between the Fourier spectrum obtained for images restored via both spatial domain low pass filters and frequency domain sym- metrically elliptical gaussian filters (Fig. 12a), are mirrored by their variance values, which are almost coincident. Figure 18 also shows that the es timated noise is remarkably high when compared to the original image itself on an absolute scale. Minimisation of its effect is, therefore, inevitably necessary.

Whereas a ZNF significantly suppresses the noise in the SWIR data, it 


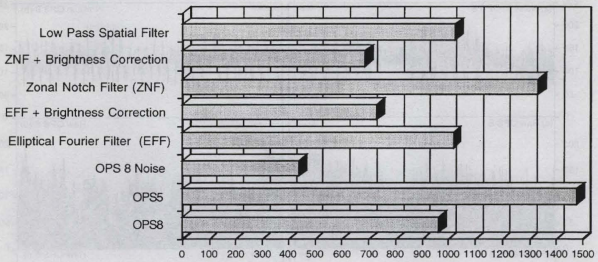

Figure 18 - Variances of a raw JERS-1 OPS8 image and variances for several restored versions of the same OPS8. Note that all images restored in the frequency domain and corrected for brightness saturation display lower variance range.

does not totally eliminate the noise. This is true for all filtering methods in which the noise and signal overlap in the spatial and spectral domains (Simpson \& Yhann, 1994). In these cases, the noise cannot be totally removed without a complete description of the noise or signal. The best that can be achieved is that the residual noise is reduced as much as possible with as little as possible image distortion introduced.

\section{ACKNOWLEDGEMENTS}

C.R. de Souza Filho acknowledges the support of Conselho Nacional de Pesquisas (CNPq-Brazil) and Fundação de Amparo a Pesquisa no Estado de São Paulo (FAPESP) through research grants 301.227/94 and 1996/11139-2, respectively. The authors would like to thank CNES and Tim Minor (Desert Research Institute - Nevada - USA) for kindly providing us with the SPOT data. We are also grateful to Takashi Nishidai (JAPEX- Tokyo - Japan) and Andrew Harris (University of Hawaii - USA) for their contribution with the JERS-1 and AVHRR data, respectively.

\section{REFERENCES}

CASTLEMAN, K.R. (1979) Digital image processing . New Jersey, Prentice Hall. 429p.

CHAVEZ JR, P.S.; KWARTENG, A.Y. (1989) Extracting spectral contrast Landsat Thematic Mapper image data using selective principal component analysis. Photogrammetric Engineering \& Remote Sensing, v.55, n.3, p. 339-348.

CRIPPEN, R.E. (1989) A simple spatial filtering routine for the cosmetic removal of Scan-Line Noise from Landsat TM P-Tape Imagery. Photogrammetric Engineering \& Remote Sensing, v.55, n. 3, p. $327-$ 331.

CRIPPEN, R.E.; FORD, J.P.; BLOM, R.G. (1994) An evaluation of Fuyo1 (JERS-1) optical data for geological interpretation. In: THEMATIC CONFERENCE ON GEOLOGICAL REMOTE SENSING, 20., San Antonio, Texas, USA, $1994 . \quad$ Proceedings. 
Michigan, Environmental Research Institute. p.606.

DAVIS, J.C. (1973) Statistics and Data Analysis in Geology. 2.ed. New York, John Wiley \& Sons. $328 \mathrm{p}$.

DE SOUZA FILHO, C.R.; DRURY, S.A.; DENNISS, A.M.; CARLTON, R.W.T.; ROTHERY, D.A. (1996) Restoration of corrupted optical Fuyo-1 (JERS-1) data using frequency domain techniques. Photogrammetric Engineering \& $\mathbf{R e}$ mote Sensing, v. 62 , n.9, p.10371047.

DRURY, S.A. (1993) Image interpretation in geology. 2.ed. London, Chapman \& Hall. 283p.

DRURY, S.A.; HUNT, G.A. (1988) Remote sensing in laterised archean terrain: Marshall Pool area, Northeastern Yilgarn Block, Western Australia. Photogrammetric Engineering \& Remote Sensing, v.54, n.4, p. 1717-1725.

ELIASON, E.M.; MCEWEN, A.S. (1990) Adaptive box filters for removal of random noise from digital images. Photogrammetric Engineering \& Remote Sensing, v.56, n. 4, p. $453-458$.

GONZALEZ, R.C.; WINTZ, P. (1977) Digital image processing. Addison-Wesley, 502p.

GREEN, A.A.; BERMAN, M; SWITZER, P.; CRAIG, M.D. (1988) A transformation for ordering multispectral data in terms of image quality with implications for noise removal. IEEE Transactions on Geoscience and Remote Sensing, v. 26 , n. 2, p. 65 74.

HUMMER-MILLER, S. (1990) Techniques for noise removal and registration of TIMS data. Photogram- metric Engineering \& Remote Sensing, v.56, n. 1, p. 49-53.

HUNT, G.A. (1991) Geological applications of reflected and emitted multispectral data over lateritised archean terrain in Western Australia. Milton Keynes, England, 290p. (Unpublished $\mathrm{PhD}$ Thesis, The Open University, UK).

LOUGHLIN, W.P. (1991) Principal component analysis for alteration mapping, Photogrammetric Engineering \& Remote Sensing, v. 57, n. 9, p. 1163-1169.

PELI, T.; LIM, J.S. (1982) Adaptive filtering for image enhancement. Optical Engineering, v. 21, n. 1, p. 108-114.

READY, P.J,; WINTZ, P.A. (1973) Information extraction, SNR improvements and data compression in multispectral imagery. IEEE Transactions Commun, v. 21, p. 1123-1132.

ROSE, J.F. (1989) Spatial Interference in the AVIRIS i maging spectrometer. Photogrammetric Engineering \& Remote Sensing, v. 55, n. 9, p.1339-1346.

ROTHERY, D.A.; HUNT, G.A. (1990) A simple way to perform decorrelation stretching and related techniques on menu-driven processing systems. International Journal Remote Sensing, v.1, p. 133-137.

SCHOWENGERDT, R.A. (1983)

Techniques for image processing and classification in remote sensing. London, Academic Press. 239 p.

SINGH, A.; HARRISON, A. (1985) Standardised principal components. International Journal Remote Sensing, v. 6, p.883-896. 
STRICKLAND, R.N.; ALY, M.Y. (1985) Imagesharpness enhancement using Adaptive $3 \times 3$ convolution masks. Optical Engineering, v. 24, n. 4 , p. 683-686.

SIMPSON, J.J.; YHANN, S.R. (1994) Reduction of noise in AVHRR Channel 3 data with minimum distortion, IEEE Transactions Geoscience Remote Sensing, v. 32, n. 2 , p. $315-328$.

TOWNSEND, J.R.G. (1984) Agriculture land-cover discrimination using thematic mapper spectral bands,
International Journal Remote Sensing, v. 5, p. 681-698.

WAREN, D. (1989) AVHRR channel-3 noise and methods for its removal. International Journal of Remote Sensing, v. 10 , p. $645-651$. WESTIN, T. (1990) Filters for removing coherent noise of period 2 in SPOT imagery. International Journal Remote Sensing, v.11, n. 2, p. 351-357.

C.R.Souza Filho - Instituto de Geociências, Universidade Estadual de Campinas, Caixa Postal .6252, CEP 13081-970, Campinas, SP, Brasil. 